\title{
Practical dosing of propofol in morbidly obese patients
}

\author{
Ban C. H. Tsui, MSc, MD $\mathbb{D} \cdot$ Luke Murtha, MBBS • Hendrikus J. M. Lemmens, MD, PhD
}

Received: 9 December 2016/Revised: 10 January 2017/ Accepted: 21 February 2017/Published online: 28 February 2017

(c) Canadian Anesthesiologists' Society 2017

The increased incidence of obesity and its associated comorbidities has had significant impact on medical practice, especially anesthesia. ${ }^{1}$ For example, diabetes, hypertension, obstructive sleep apnea, and cardiopulmonary disease all decrease the margin of safety for anesthetic agents in morbidly obese (MO) patients. Morbid obesity (defined as a body mass index $[\mathrm{BMI}]>40$ $\mathrm{kg} \cdot \mathrm{m}^{-2}$ ) has been shown to have a significant effect on the metabolism and pharmacokinetic profiles of drugs used for the induction of anesthesia. This leads to challenges in accurate estimation of dosage requirements for $\mathrm{MO}$ patients.

\section{Pharmacokinetic considerations}

Four major factors ${ }^{1}$ have been identified that affect the pharmacokinetics and pharmacodynamics of anesthetic agents in MO patients: 1) increased cardiac output (CO), 2) increased lean body weight (LBW), 3) increased fat mass, and 4) increased extracellular fluid volume.

Increased circulation due to increased $\mathrm{CO}$ leads not only to more rapid onset and offset of drug effects but also to lower peak plasma concentration and shorter duration of effect. Lean body weight, which is equal to total body weight (TBW) minus body fat weight (BFW), is where

B. C. H. Tsui, MSc, MD $(\bowtie) \cdot$ H. J. M. Lemmens, MD, PhD Department of Anesthesiology, Perioperative and Pain Medicine, Stanford University, Palo Alto, CA, USA

e-mail: bantsui@stanford.edu

L. Murtha, MBBS

Department of Anesthesiology and Pain Medicine, University of Alberta, Edmonton, AB, Canada most metabolism occurs and is positively correlated to drug clearance rates.

In MO patients, the ratio of BFW/TBW is increased, while the ratio of LBW/TBW is decreased; however, there is an overall increase in both LBW and BFW. ${ }^{1,2}$ Higher cardiac output and LBW indicate that obese patients may generally require larger doses of propofol than non-obese patients; however, these doses are still less than those calculated based on their TBW.

Increased fat mass in MO patients can lead to a larger volume of distribution for lipophilic drugs such as propofol; however, the actual pharmacokinetic effect of this is tempered and complicated by the fact that there is generally a reduced blood flow to adipose tissue. More drug may therefore be delivered to non-adipose tissue in the acute induction phase, resulting in higher concentrations in those areas. Lower doses of propofol may be required during the induction phase to compensate for this transient period of reduced distribution. ${ }^{1}$

Last, but not least, is the effect of increased extracellular fluid volume. The water content of adipose tissue is almost completely extracellular. Extracellular fluid volume and the extracellular/intracellular fluid volume ratio are increased in MO patients. This can result in an increased volume of distribution for hydrophilic substances such as muscle relaxants, but it does not have as much effect on highly lipophilic drugs like propofol..$^{1,3}$

\section{Optimal induction dose of propofol}

Use of LBW as a weight-based scalar

Of interest, propofol is one of the most commonly used drugs for the induction and maintenance of general 
anesthesia in modern anesthetic practice. While bolus dose recommendations for this powerful agent based on TBW are valid in patients with normal BMI, they can be dangerously problematic in MO subjects due to their altered pharmacokinetic profiles. Despite these differences, propofol is still often dosed in this manner for the MO population because of its ease in clinical practice. Thus, it is desirable and necessary to find a simple and practical weight-based scalar for optimal dosing of propofol in $\mathrm{MO}$ patients-i.e., a scalar that will allow for fast induction times and less hemodynamic instability. ${ }^{4}$

Lean body weight has been seen as a promising option not only because of its strongly linear correlation to both cardiac output ( $\mathrm{CO}$ ) and drug clearance but also because it negates the need to account for the reduced level of blood flow to the large amount of adipose tissue in MO patients. ${ }^{1}$ One prospective study by Ingrande et $a{ }^{4}{ }^{4}$ found that anesthesia induction in MO subjects with a propofol infusion based on LBW (measured by bioelectrical impedance analysis) produced results similar to anesthesia induction in non-obese control subjects given a propofol infusion based on TBW. The implication of this study's results is that LBW is appropriate for dosing propofol to achieve loss of consciousness in MO patients, while TBW is suitable for non-obese patients. ${ }^{4}$ Nevertheless, problems may arise in resolving how to draw the line between MO and non-obese patients when determining whether to use LBW or TBW. In light of this quandary, alternative dosing regimens modified from LBW (e.g., lean-scaled weights) ${ }^{5}$ have also been proposed but have not been validated clinically.

Use of neuromonitoring devices as a physiological guide

In this issue of the Journal, Subramani et al. ${ }^{6}$ examined whether an induction dose of propofol based on a bispectral index (BIS) target was superior to an induction dose based on LBW at achieving loss of consciousness in MO patients. The authors compared two methods estimating depth of anesthesia-i.e., a nonscalar method utilizing a brain function monitoring device (BIS) and a scalar method based on an estimation of LBW. Patients were randomized into two groups ( $n=30$ per group) and received propofol infusions of $100 \mathrm{mg} \cdot \mathrm{kg}^{-1} \cdot \mathrm{hr}^{-1}$ to initial target endpoints based on either a BIS of 50 (BIS group) or a pre-calculated dose of $2.6 \mathrm{mg} \cdot \mathrm{kg}^{-1} \mathrm{LBW}$ (LBW group). ${ }^{6}$

The level (or depth) of anesthesia in each group was then determined using the Observer's Assessment of Alertness/Sedation (OAA/S) scale, where a lack of response to a painful trapezius squeeze signified a score of 0 , which was considered to indicate loss of consciousness (LOC). If a score of 0 was not achieved at the target endpoint, the infusion was continued and the dose required to achieve a score of 0 was observed. ${ }^{6}$

The reported result of this study showed that, in MO patients, the induction dose of propofol based on BIS was different from the induction dose based on LBW. This in itself is arguably an ambiguous finding as it is only logical that two different methods produce two different results. Nevertheless, most pertinent to this present editorial is the fact that 18 of the 30 patients $(60 \%)$ in the LBW group required additional propofol to achieve an OAA/S score of 0 , essentially casting doubt on the utility of LBW as a scalar for accurate estimation of propofol dosing in $\mathrm{MO}$ patients. ${ }^{6}$

\section{Use of LBW vs BIS}

At first glance, these results may appear contrary to those reported by Ingrande et $a{ }^{4}{ }^{4}$ concerning the usefulness of LBW-based propofol dosing. Nevertheless, there are subtle but critical differences in the studies that may account for these disagreeing results. Two important concepts in clinical anesthesia can be highlighted by these differences, i.e., "depth" of anesthesia and "balance" of anesthesia.

\section{"Level" of anesthesia}

There are four levels of sedation and anesthesia, namely, minimal sedation, moderate sedation, deep sedation, and general anesthesia. The way in which the level of sedation or anesthesia was assessed differed between the two studies.

In the study by Ingrande et al., ${ }^{4}$ each subject was instructed to hold a $20-\mathrm{mL}$ syringe between the thumb and index finger while lying on a table in a quiet operating room. Loss of consciousness was determined when the patient dropped the syringe. Although not reported explicitly in that article, this definition of LOC appears to resemble moderate or deep sedation, which would correspond to an OAA/S score of $\leq 2$. In contrast, in the study by Subramani et al., an OAA/S score of 0 indicated confirmation of LOC, which would correspond to a level consistent with general anesthesia. This difference in endpoints-i.e., initial moment of relaxation when losing consciousness in a quiet room $v s$ lack of response to a painful stimulus-clearly indicates the significantly different levels of anesthesia attained in the two studies. In fact, previous investigators, including some of the primary references ${ }^{7}$ in Subramani et al., have considered an OAA/S score of $\leq 2$ to be "sufficient depth of anesthesia" to indicate LOC. Without providing any rationalization, Subramani et al. ${ }^{6}$ chose a much deeper 
level of anesthesia as their target and, in doing so, appear to have transformed the primary endpoint of their study from loss of consciousness (deep sedation) to loss of pain sensation (general anesthesia).

"Balanced" anesthesia

In modern practice, "balanced" anesthesia ${ }^{8}$ refers to the administration of combinations of several anesthetic agents in order to maximize the advantages, but not the disadvantages, of each individual component. For example, typical regimens can include both a hypnotic (e.g., propofol) to induce LOC and an opiate (e.g., fentanyl) to blunt the response to noxious stimuli. The timing of administration of these individual agents is critical in order to achieve the desired effect. The importance of this point is well illustrated when examining the differences in protocol between these same two studies.

In the study by Ingrande et al. ${ }^{4}$ fentanyl was given only after LOC had been established due to the effect of the propofol infusion, allowing the authors to avoid any confounding of results with the effects of a second drug. In contrast, in the study by Subramani et al., fentanyl was given prior to the propofol infusion. This administration of an opiate makes it difficult to be sure if LOC-defined in this study as a lack of response to painful stimuli-was due to the effect of propofol alone or to a combination effect of the fentanyl and propofol. Fentanyl's peak effect occurs approximately three to five minutes after administration ${ }^{2}$; therefore, both the BIS and LBW groups could have become unresponsive to the trapezius squeeze mostly due to the clinical effect of fentanyl rather than to true anesthesia induced by propofol alone.

In the design framework of an ideal study concerning the LOC effect of propofol, it would have been more appropriate to delay the fentanyl administration until after LOC had been achieved, as described in Ingrande et al. ${ }^{4}$ Otherwise, the study, as with Subramani et al., ${ }^{6}$ can be said to examine only the combination effect of two induction agents (propofol and fentanyl), not one agent in isolation. The collected results may reflect these limitations in the study design; therefore, they should be treated with caution.

In clinical practice, however, it is routine to give fentanyl two to three minutes prior to an induction dose of propofol in order to mitigate the adverse physiologic consequences of intubation. Caution should be taken not to let such important decision-making become automatic. We hope the example presented by these studies can act as a reminder to clinicians regarding the concept of balanced anesthesia and the importance of careful consideration of the onset times and pharmacokinetic profiles of individual drugs when used in combination.

\section{Complexity in calculating LBW}

Because of the potential of new technologies, such as BIS, estimations based on scalars may well become obsolete in the near future. Nevertheless, until then, it is important that we strive for the most accurate ways of estimating doses in patients of all types. As such, LBW should be considered a useful scalar for dosing propofol in obese patients. While most experts consider the Janmahasatian equation ${ }^{9}$ to be the most accurate way to estimate LBW, many find it difficult to use without a medical formula calculator. Errors can be made not only when performing the calculation but also when simply writing out the complex equation. An inadvertent omission of any set of brackets could drastically alter the result of the calculation and potentially lead to grossly and dangerously misestimated LBW and hence propofol dosing. Thus, further research will be needed to develop an accurate but simplified equation model suitable for practical use.

Despite these issues, the authors should be commended for their painstaking and well-written article illustrating an important and complex pharmacological interaction. The value of real-time neuromonitoring (such as BIS or a similar processed electroencephalogram monitor) that can be titrated to effect should not be understated and is wholly deserving of study. In theory, this has the potential to provide effective monitoring and control of depth of anesthesia, better hemodynamic stability, and faster recovery with improved patient comfort, both in nonobese and obese patients receiving general anesthesia. ${ }^{10}$ Nevertheless, while it is important to point out that the final induction dose should be carefully titrated to achieve desired clinical effect (regardless of the weight scalar used) for the initial estimated dosing, many experts consider LBW to be an improvement over TBW and use it preferentially when dosing propofol for induction of anesthesia in MO patients.

\section{Posologie pratique du propofol chez des patients présentant une obésité morbide}

L'augmentation de l'incidence de l'obésité et des comorbidités qui lui sont associées a eu un impact significatif sur la pratique médicale et, en particulier, sur l'anesthésie. ${ }^{1}$ Par exemple, diabète, hypertension, syndrome d'apnée-hypopnée du sommeil et maladie 
cardiopulmonaire réduisent tous la marge de sécurité des agents anesthésiques chez les patients présentant une obésité morbide $(\mathrm{OM})$. On a montré que l'OM (définie par un indice de masse corporelle [IMC] $>40 \mathrm{~kg} \cdot \mathrm{m}^{-2}$ ) avait un effet important sur le métabolisme et les profils pharmacocinétiques des médicaments utilisés pour l'induction de l'anesthésie. Cela crée des défis pour l'estimation des doses exactes requises par les patients présentant une OM.

\section{Considérations pharmacocinétiques}

Quatre principaux facteurs ${ }^{1}$ modifiant la pharmacocinétique et la pharmacodynamie des agents anesthésiques chez les patients présentant une $\mathrm{OM}$ ont été identifiés: 1) augmentation du débit cardiaque (DC), 2) augmentation du poids de la masse corporelle maigre (MCM), 3) augmentation de la masse grasse, et 4) augmentation du volume liquidien extracellulaire.

L'augmentation de la circulation sanguine causée par l'augmentation du DC aboutit non seulement à une apparition et disparition plus rapides de l'effet des médicaments, mais aussi à une baisse des concentrations plasmatiques maximum et à des durées d'action plus courtes. La masse maigre qui est égale au poids corporel total (PT) moins le poids de la masse corporelle grasse (MCG) est l'endroit où se déroule l'essentiel du métabolisme; cette masse maigre est positivement corrélée aux vitesses d'épuration des médicaments.

Chez les patients présentant une OM, le rapport MCG/ PT est augmenté, alors que le rapport MCM/PT est diminué; il existe toutefois une augmentation globale de la MCM et de la MCG. ${ }^{1,2}$ Un débit cardiaque et une MCM augmentés indiquent que les patients obèses auront habituellement besoin de plus grandes doses de propofol que les patients non obèses; toutefois, ces doses sont inférieures à celles calculées en fonction de leur PT.

L'augmentation de la masse grasse chez les patients ayant une OM peut entraîner un plus grand volume de distribution des médicaments lipophiles tels que le propofol, mais ce véritable effet pharmacocinétique est tempéré et compliqué par le fait que le débit sanguin est habituellement réduit dans le tissu adipeux. Il est donc possible que davantage de médicaments soient délivrés dans le tissu non adipeux au cours de la phase d'induction aiguë, entraînant des concentrations plus importantes dans ces secteurs. Des doses moindres de propofol pourraient être requises pendant la phase d'induction pour compenser cette période transitoire de distribution réduite. ${ }^{1}$

Enfin, et ce n'est pas le moindre, il y a l'effet de l'augmentation du compartiment liquidien extracellulaire. Le contenu en eau du tissu adipeux est presque complètement extracellulaire. Le volume liquidien extracellulaire et le rapport du volume liquidien extracellulaire/intracellulaire sont augmentés chez les patients présentant une OM. Cela peut se traduire par une augmentation du volume de distribution des substances lipophiles, telles que les relaxants musculaires, mais n'a pas autant d'effet que sur des médicaments fortement lipophiles comme le propofol. ${ }^{1,3}$

\section{Dose d'induction optimale du propofol}

Utilisation de la MCM comme scalaire basé sur le poids

Il est intéressant de noter que le propofol est l'un des médicaments le plus souvent utilisé pour l'induction et l'entretien de l'anesthésie générale en pratique anesthétique moderne. Alors que les recommandations posologiques basées sur le PT concernant les bolus restent valides pour ce produit puissant chez les patients à l'IMC normal, elles peuvent devenir dangereusement problématiques chez les patients ayant une $\mathrm{OM}$ en raison de l'altération de leurs profils pharmacocinétiques. Malgré ces différences, le propofol est encore souvent dosé de cette manière pour la population atteinte d'OM en raison de sa commodité en pratique clinique. Il est donc souhaitable et nécessaire de trouver un scalaire simple et pratique, basé sur le poids, pour une administration de la posologie optimale de propofol à des patients ayant une $\mathrm{OM}$, c'est-à-dire, une échelle qui permettra des temps d'induction courts et une moindre instabilité hémodynamique. ${ }^{4}$

On a considéré que le poids de la masse corporelle maigre était une option prometteuse, non seulement en raison d'une forte corrélation linéaire avec le débit cardiaque (DC) et l'élimination des médicaments, mais aussi parce qu'elle ignore le besoin de tenir compte du débit sanguin réduit dans la grande masse de tissu adipeux des patients présentant une obésité morbide. ${ }^{1}$ Dans leur étude prospective, Ingrande et coll. ${ }^{4}$ ont trouvé que l'induction de l'anesthésie chez des patients présentant une $\mathrm{OM}$ avec une perfusion de propofol dosée en fonction de la MCM (mesurée par l'analyse de l'impédance bioélectrique) entraînait des résultats semblables à l'induction d'une anesthésie chez des patients-contrôles non obèses recevant une perfusion de propofol basée sur leur PT. Les résultats de cette étude impliquent que la MCM permet le calcul correct de la posologie du propofol entraînant la perte de conscience des patients ayant une $\mathrm{OM}$ et que le PT convient pour les patients non obèses. ${ }^{4}$ Néanmoins, des problèmes peuvent surgir quand, pour savoir s'il faut utiliser la MCM ou le PT, il faut établir la limite entre patients ayant une $\mathrm{OM}$ et patients non obèses. 
Devant ce dilemme, des schémas posologiques modifiés sur la base du poids de la masse maigre (par exemple, poids pondéré par la masse maigre) $)^{5}$ ont aussi été proposés, mais n'ont pas été validés en clinique.

Utilisation des dispositifs de monitorage neurologique comme guide physiologique

Dans ce numéro du Journal, Subramani et coll. ${ }^{6}$ ont voulu savoir si une dose d'induction de propofol basée sur un indice bispectral (BIS) cible était plus efficace qu'une dose basée sur le poids de la masse maigre pour entraîner la perte de conscience chez des patients présentant une OM. Les auteurs ont comparé deux méthodes d'estimation de la profondeur de l'anesthésie, c'est-à-dire une méthode non scalaire utilisant un dispositif de monitorage des fonctions neurologiques (BIS) et une méthode scalaire basée sur une estimation de la masse maigre. Les patients ont été randomisés dans deux groupes ( $n=30$ par groupe) et ont reçu des perfusions de propofol à raison de $100 \mathrm{mg} \cdot \mathrm{kg}^{-1} \cdot \mathrm{h}^{-1}$ jusqu'à l'obtention des critères d'évaluation cibles initiaux, en fonction d'un BIS de 50 (groupe «BIS») ou d'une dose précalculée de $2,6 \mathrm{mg} \cdot \mathrm{kg}^{-1} \mathrm{MCM}$ (groupe « MCM »). ${ }^{6}$

Le niveau (ou profondeur) de l'anesthésie dans chaque groupe a été établi au moyen de l'échelle OAA/S (évaluation par l'observateur de l'éveil/sédation) dans laquelle une absence de réponse à un pincement douloureux du trapèze équivaut à un score de 0 , jugé comme indiquant une perte de conscience (PC). Si le score de 0 n'était pas atteint au moment du critère d'évaluation cible, la perfusion était poursuivie et la dose requise pour l'obtention du score de 0 était consignée. ${ }^{6}$

Les résultats rapportés de cette étude ont montré que chez les patients présentant une OM, la dose d'induction du propofol basée sur le BIS était différente de la dose d'induction basée sur le poids de la MCM. Ceci est en soi une constatation possiblement ambiguë, car il est logique que deux méthodes différentes parviennent à des résultats différents. Néanmoins, le fait que 18 des 30 patients $(60 \%)$ du groupe MCM aient eu besoin d'une dose supplémentaire de propofol pour parvenir au score 0 de l'échelle OAA/S est plus pertinent pour cet éditorial, jetant un doute sur l'utilité de la MCM comme base de calcul d'une estimation précise de la posologie de propofol requise par les patients présentant une OM. ${ }^{6}$

\section{Utilisation de la MCM ou du BIS}

À première vue, ces résultats peuvent paraîtres contradictoires avec ceux rapportés par Ingrande et coll. ${ }^{4}$ sur l'utilité d'une administration de propofol reposant sur le poids de masse maigre. Il existe cependant des différences subtiles, mais essentielles entre les études qui pourraient expliquer ces résultats discordants. Deux importants concepts d'anesthésie clinique peuvent être soulignés par ces différences: la «profondeur» et «l'équilibre » de l'anesthésie.

« Profondeur » de l'anesthésie

Il y a quatre niveaux de sédation et d'anesthésie: sédation minime, sédation modérée, sédation profonde et anesthésie générale. La façon dont le niveau de sédation ou d'anesthésie a été évalué était différente dans ces deux études.

Dans l'étude d'Ingrande et coll., ${ }^{4}$ les patients ont eu comme consigne de tenir une seringue de $20 \mathrm{~mL}$ entre le pouce et l'index alors qu'ils étaient allongés sur une table dans une salle d'opération calme. La perte de conscience était définie par le moment où le patient laissait tomber la seringue. Bien que cela ne soit pas décrit de façon explicite dans cet article, la définition de perte de conscience ressemble à celle d'une sédation modérée ou profonde qui correspondrait à un score $\mathrm{OAA} / \mathrm{S}$ de $\leq 2$. En revanche, dans l'étude de Subramani et coll., un score OAA/S de 0 indiquait une perte de conscience confirmée dont le niveau correspondrait à une anesthésie générale. Cette différence sur les critères d'évaluation - c' est-à-dire, le moment initial de relaxation avec la perte de conscience dans une pièce calme opposé à l'absence de réponse à un stimulus douloureux - indique clairement les différents niveaux d'anesthésie atteints par les deux études. En fait, de précédents investigateurs, y compris quelquesuns figurant parmi les principales références ${ }^{7}$ de Subramani et coll. ont considéré qu'un score OAA/S $\leq 2$ était une "profondeur suffisante d'anesthésie » pour indiquer la perte de conscience. Sans fournir aucune justification, Subramani et coll. ${ }^{6}$ ont choisi un niveau d'anesthésie beaucoup plus profond que leur cible et en faisant cela, ils semblent avoir transformé le critère d'évaluation principal de leur étude d'une perte de conscience (sédation profonde) à la perte de la sensation douloureuse (anesthésie générale).

Anesthésie « équilibrée »

Dans la pratique actuelle, une anesthésie «équilibrée ${ }^{8}$ fait référence à l'administration d'une combinaison de plusieurs agents anesthésiques dans le but d'optimiser les bénéfices, mais pas les inconvénients, de chaque molécule prise individuellement. Les protocoles habituels peuvent inclure, par exemple, un hypnotique (par ex., le propofol) pour induire la perte de conscience et un opiacé (par ex., le fentanyl) pour émousser la réponse aux stimuli douloureux. 
Le moment de l'administration de chacune de ces molécules est essentiel pour obtenir l'effet désiré. L'importance de ce point est bien illustrée par l'examen des différences entre les protocoles de ces deux mêmes études.

Dans la série d'Ingrande et coll., ${ }^{4}$ le fentanyl n'a été administré qu'après la confirmation de la perte de conscience sous l'effet de la perfusion de propofol, ce qui a permis aux auteurs d'éviter tout facteur confondant lié aux effets du deuxième médicament sur les résultats. Dans l'étude de Subramani et coll., le fentanyl a été administré avant la perfusion de propofol. Il devient difficile avec l'administration de cet opiacé d'avoir la certitude que la perte de conscience - définie dans l'étude comme une absence de réponse à un stimulus douloureux - résulte de l'effet du propofol seul ou d'un effet combiné du fentanyl et du propofol. L'effet maximum du fentanyl apparaît environ trois à cinq minutes après son administration $^{2}$; les deux groupes (BIS et MCM) pourraient donc avoir cessé de répondre au pincement du trapèze principalement à cause du fentanyl plutôt qu'en raison d'une véritable anesthésie induite par le seul propofol.

Il aurait été préférable, dans la conception d'une étude idéale sur l'effet de perte de conscience du propofol, de retarder l'administration du fentanyl après l'obtention de la perte de conscience, comme décrit dans l'étude d'Ingrande et coll. ${ }^{4}$ Sinon, comme dans le cas de l'étude de Subramani et coll., ${ }^{6}$ on peut dire que l'étude n'examine que l'effet combiné de deux agents d'induction (le propofol et le fentanyl) et pas d'un seul de ces produits pris séparément. Les résultats collectés pourraient refléter les limites du plan de l'étude et, par conséquent, ils doivent être traités avec prudence.

En pratique clinique, il est toutefois usuel d'administrer le fentanyl deux à trois minutes avant la dose d'induction de propofol afin de réduire les conséquences physiologiques indésirables de l'intubation. Il faut veiller à ce qu'une prise de décision aussi importante ne devienne pas automatique. Nous espérons que les exemples présentés dans ces études serviront de rappel aux cliniciens sur le concept d'anesthésie équilibrée et sur l'importance à accorder aux délais d'action et aux profils pharmacocinétiques des différents médicaments quand ils sont utilisés ensemble.

\section{Le calcul du poids de la masse maigre: complexe}

Les estimations basées sur des scalaires pourraient bien devenir obsolètes dans un proche avenir en raison de l'arrivée potentielle de nouvelles technologies comme l'indice bispectral. Mais, en attendant, il est important que nous nous efforcions de trouver des moyens plus précis pour estimer les doses pour tous les types de patients. Dans ces conditions, le poids de la masse maigre (MCM) doit être considéré comme un scalaire utile pour le dosage du propofol chez les patients obèses. La majorité des experts estiment que l'équation de Janmahasatian' ${ }^{9}$ est le moyen d'estimer le plus exactement possible la MCM, mais nombreux sont ceux qui la trouvent difficile à utiliser sans une calculatrice de formules médicales. Il est possible de faire des erreurs non seulement en effectuant le calcul, mais aussi simplement en écrivant cette équation complexe. L'omission accidentelle de la moindre parenthèse pourrait modifier totalement le résultat du calcul et aboutir potentiellement à une estimation grossièrement et dangereusement erronée de la masse maigre, et donc de la posologie du propofol. Par conséquent, des recherches supplémentaires sont nécessaires pour élaborer un modèle d'équation précise, mais simplifiée, utilisable en pratique.

Malgré ces problèmes, les auteurs doivent être félicités pour leur article difficile et bien écrit qui illustre une interaction pharmacologique importante et complexe. La valeur du monitorage neurologique en temps réel, tel que le BIS ou un moniteur similaire basé sur l'EEG, qui permet d'augmenter les doses jusqu'à l'obtention de l'effet souhaité ne doit pas être sous-estimé et justifie pleinement d'être étudié. Cela pourrait, en théorie, fournir un mode de surveillance et un contrôle efficaces de la profondeur de l'anesthésie, une meilleure stabilité hémodynamique et une récupération plus rapide des patients avec l'amélioration de leur confort, qu'ils soient obèses ou non, lorsqu'ils reçoivent une anesthésie générale. $^{10}$ Mais bien qu'il soit important de souligner que la dose d'induction définitive doit être augmentée prudemment pour parvenir à l'effet clinique désiré (indépendamment de la référence de poids utilisée) pour la première administration, de nombreux experts estiment que la MCM est un progrès par rapport au poids total et l'utilisent préférentiellement quand ils administrent du propofol pour l'induction de l'anesthésie chez des patients présentant une OM.

Conflicts of interest None declared.

Editorial responsibility This submission was handled by Dr. Hilary P. Grocott, Editor-in-Chief, Canadian Journal of Anesthesia.

Funding sources None.

Other associations None.

Conflits d'intérêts Aucun déclaré.

Responsabilité éditoriale Cet article a été traité par le $\mathrm{D}^{\mathrm{r}}$ Hilary $\mathrm{P}$. Grocott, Rédacteur en chef, Journal canadien d'anesthésie. 
Sources de financement Aucune.

Autres associations Aucune.

\section{References}

1. Lemmens HJ. Perioperative pharmacology in morbid obesity. Curr Opin Anaesthesiol 2010; 23: 485-91.

2. Ingrande J, Lemmens HJ. Dose adjustment of anaesthetics in the morbidly obese. Br J Anaesth 2010; 105(Suppl 1): i16-23.

3. Baerdemaeker LE, Mortier EP, Struys MM. Pharmacokinetics in obese patients. Contin Educ Anaesth Crit Care Pain 2004; 4: 1525.

4. Ingrande J, Brodsky JB, Lemmens HJ. Lean body weight scalar for the anesthetic induction dose of propofol in morbidly obese subjects. Anesth Analg 2011; 113: 57-62.

5. Friesen JH. Lean-scaled weight: a proposed weight scalar to calculate drug doses for obese patients. Can J Anesth 2013; 60: 214-5.
6. Subramani Y, Riad W, Chung F, Wong J. Optimal propofol induction dose in morbidly obese patients: A randomized controlled trial comparing bispectral index and lean body weight scalar. Can J Anesth 2017; 64: this issue. DOI: 10.1007/ s12630-017-0852-x

7. Gurses E, Sungurtekin H, Tomatir E, Dogan H. Assessing propofol induction of anesthesia dose using bispectral index analysis. Anesth Analg 2004; 98: 128-31.

8. Tonner PH. Balanced anaesthesia today. Best Pract Res Clin Anaesthesiol 2005; 19: 475-84.

9. Janmahasatian S, Duffull SB, Ash S, Ward LC, Byrne NM, Green $B$. Quantification of lean bodyweight. Clin Pharmacokinet 2005; 44: 1051-65.

10. Pandazi A, Bourlioti A, Kostopanagiotou G. Bispectral index (BIS) monitoring in morbidly obese patients undergoing gastric bypass surgery: experience in 23 patients. Obes Surg 2005; 15: 58-62. 